Revue

Revue de l'histoire des religions

de Ihistoire des religions

Jacques LE BRUN, Dieu un pur rien. Angelus Silesius: poésie, métaphysique et mystique

Paris, Éditions du Seuil (« La librairie du XxI ${ }^{\mathrm{e}}$ siècle »), 2019

Sophie Houdard

\title{
OpenEdition
}

Journals

Édition électronique

URL : http://journals.openedition.org/rhr/11099

DOI : $10.4000 /$ rhr. 11099

ISSN : 2105-2573

Éditeur

Armand Colin

Édition imprimée

Date de publication : 1 mars 2021

Pagination: 160-162

ISBN : 978-2-200-93375-3

ISSN : 0035-1423

Référence électronique

Sophie Houdard, « Jacques Le Brun, Dieu un pur rien. Angelus Silesius : poésie, métaphysique et mystique », Revue de l'histoire des religions [En ligne], 1 | 2021, mis en ligne le 19 mars 2021, consulté le 31 mars 2021. URL : http://journals.openedition.org/rhr/11099; DOI : https://doi.org/10.4000/rhr. 11099

Ce document a été généré automatiquement le 31 mars 2021.

Tous droits réservés 


\section{Jacques LE BRUN, Dieu un pur rien. Angelus Silesius : poésie, métaphysique et mystique}

Paris, Éditions du Seuil (« La librairie du XxI siècle »), 2019

\section{Sophie Houdard}

\section{RÉFÉRENCE}

Jacques LE BRUn, Dieu un pur rien. Angelus Silesius : poésie, métaphysique et mystique, Paris, Éditions du Seuil (« La librairie du XxI ${ }^{\mathrm{e}}$ siècle »), 2019, 240 p., 22,5 cm, $21 €$, ISBN

978-2-02-113947-1.

1 Derrière ce titre épuré se cache une lecture rigoureuse des enjeux mystiques et métaphysiques des distiques et quatrains qui composent Le Pèlerin chérubinique que Johannes Scheffler publie une première fois à Vienne en 1657 sous le nom d'Angelus Silesius. Jacques Le Brun nous donne ici une interprétation du recueil en travaillant le rapport entre une culture spirituelle très dense et une écriture poétique d'une brièveté intense, rapport que saisit l'énoncé, qui donne ici le titre du livre, Dieu est un pur rien, qui cite le distique I, 25, pour poser « la difficile question du rien, point crucial de la théologie négative et sans doute de toute mystique » (p. 39). Très grand connaisseur du poète, Jacques Le Brun (qui rend ici hommage à Jean Orcibal qui le lui a fait connaître en 1953) l'est aussi de la langue et de la mystique «baroque » allemandes, au sens où Walter Benjamin employait ce terme pour désigner au cœur du drame silésien du $\mathrm{XVII}^{\mathrm{e}}$ siècle, le Trauerspiel, une incurable mélancolie et une perte irréparable. Car Johannes Scheffler, comme l'indique le prologue de l'ouvrage, est « un Silésien en des temps tragiques ». Les strophes courtes où dominent antithèses et paradoxes découpent les formes d'une mystique et d'une métaphysique d'une intense fulgurance au moment où la région jusque-là relativement tolérante aux spiritualités et confessions rivales, s'enfonce dans les dévastations de la Guerre de Trente ans. 
2 Johannes Scheffler, jeune luthérien, formé à Strasbourg, à Leyde, puis à Padoue (où il devient médecin en 1648), ami de Frankenberg et disciple de Jacob Boehme, lit Weigel, annote les mystiques rhéno-flamands et ceux $d u$ xvI ${ }^{\mathrm{e}}$ siècle, dont il se fait le "récapitulateur» (p.13) du vocabulaire de la mystique essentielle. Converti au catholicisme vers 1653 et emporté dans la virulence de la Contre-Réforme triomphante, Scheffler ne renie pourtant pas ses tendances mystiques ni ne renonce à l'« audace »terme très présent sous la plume de Jacques Le Brun - d'une métaphysique peu recevable alors dans les confessions hostiles. «En effet l'œuvre d'Angelus Silesius est en parfaite harmonie avec l'évolution profonde et souvent tragique de l'histoire de la Silésie» (p. 9). Né luthérien à Breslau (aujourd'hui Wrocław en Pologne) en 1624, revenu à un catholicisme de combat, Johannes Scheffler, ordonné prêtre en 1661, ne reniera cependant rien de la hardiesse de son Cherubinischer Wandersmann auquel il ajoutera en 1675 un sixième livre, ni ne renoncera au dessein qui était le sien en prenant le nom d'ange pour contempler et se faire le « messager » ou « l'annonciateur » (p. 8) d'une mystique essentielle qui "pousse jusqu'à l'extrême la positivité contenue dans le rien » : Dieu, comme le montre dans des pages décisives Jacques Le Brun est une chose (ein Ding) qui est un rien, voire un super-rien (ein übernichts) (p. 41). Conscient des risques d'une lecture qui confondrait un Dieu qui ne serait rien avec un Dieu qui ne serait pas (p. 42), Scheffler multiplie les atténuations et explications, dont l'expression adressée au lecteur « comprends-moi bien » (p. 162) est la formulation la plus explicite. En saisissant la force et la difficulté d'un vocabulaire souvent difficilement traduisible (comme ce verbe Entbilden pour " désimager », p. 92) ou qui nécessite un détour par les traditions bibliques, dionysiennes et rhéno-flamandes, Jacques Le Brun suit le travail de la poésie qui permet de penser (c'est-à-dire d'écrire, de produire) le dépassement, l'excès métaphysique, hors du cadre de la théologie, par une action poétique sur le langage, ce qu'une note vient adoucir ici et là sans résorber le paradoxe qui " pourrait heurter l'orthodoxie théologique" (p. 87). Ainsi, l'humanité, die Menschheit, doit s'élever au-dessus d'elle-même, et si une note vient faire du Christ, en bonne doctrine, le but suprême de l'homme, elle laisse un espace pour penser un excès, un au-delà, comme ici la «surangélicité » (die Über Engelheit) (p. 89). S'il faut réduire la radicalité de l'idée d'être Dieu, Scheffler - et Jacques Le Brun avec lui - suit la connaissance de Dieu dans l'au-delà de toute connaissance, dans le non-être, idée qu'on trouve chez Maître Eckhart, l'une des sources principales et souterraines du poète comme de la mystique protestante allemande. En suivant la signification des notions qui structurent ce voyage (la déité, la déification, l'essence, le fond, l'abîme, etc.), Jacques Le Brun montre le poids des vocabulaires mystiques dont l'histoire, les censures et les appropriations constituent le cœur de la configuration mystique.

3 C'est ce travail de reprise et de germanisation, de réusage et de déplacement que Jacques Le Brun repère et relie aux grands mystiques (le dominicain Eckhart, souvent reçu par l'intermédiaire de Jean Tauler et de la Teologia Deutsch, Ruysbroeck, Harphius) et au passeur qu'a été Sandaeus (cet autre allemand, Maximilien van der Sandt) dont la Pro theologia mystica Clavis (Cologne 1640) largement annotée par Scheffler, constitue la source lexicale et le point de départ d'un enrichissement impressionnant. Il faut lire les pages que Jacques Le Brun consacre par exemple au délaissement, la Gelassenheit si présente chez Eckhart, qu'il propose de traduire par «l'abandon de soi ", noué à la "grande hardiesse de renoncer à Dieu» (p.163) retrouvant ici la supposition impossible des mystiques qui leur fait préférer l'Enfer s'il permet de sauver l'amour pur. Les manières de dire (le modus loquendi de Sandaeus) comme les modalités du sujet 
face à ce qui est dit permettent au poète de désamorcer le risque de l'hétérodoxie dans des passages à la limite dont Jacques Le Brun fait surgir la puissance: ainsi la paradoxale dépendance de Dieu, et l'idée d'une soif essentielle dans la déité où le mot de Not (détresse) rime avec Gott (Dieu) soulignant le besoin que Dieu a de l'homme et le paradoxe fondamental que «Dieu ne peut vivre sans l'homme» d'origine encore une fois eckhartienne (p. 65).

Les «trois siècles de lecture " qui suivent montrent que la réception du Pèlerin chérubinique a affirmé, contesté ou exalté la dimension philosophique du recueil. De Leibniz qui y reconnaissait "un athéisme spéculatif caché», Schopenhauer, Kołakowski, Heidegger, Maurice Blanchot, Roger Munier, Lacan, à Jacques Derrida, c'est surtout la traduction et l'interprétation de Roger Munier parues en 1970 qui retiennent l'attention parce que Munier décontextualise le recueil poétique du Silésien pour mettre au jour une « interprétation philosophique ». Jacques Le Brun reconnaît dans ce déplacement une intuition riche qui décèle chez Angelus Silesius la mystique comme expérience noétique (ce que soulignera Jean Baruzi), dans une expérience de pensée dépouillée, purifiée des appartenances dogmatiques et confessionnelles, poétique et mystique. "Dieu, la déité, aborder le discours sur Dieu par ce qui est en Dieu et audessus de Dieu et positive négation de Dieu, seuls la poésie et un verbe d'une tragique flamboyance pouvaient tenter cette entreprise hardie et impossible; ce fut celle de Johannes Scheffler » (p. 54) : le Pèlerin chérubinique est le dernier vestige d'une union de la métaphysique et de la poésie, avant l'éclatement des trois éléments (p. 215) dont Jacques Le Brun tisse dans ce livre le rapport et le réseau dense et complexe. La poésie, la mystique et la métaphysique se séparent dès la fin du XvII ${ }^{\mathrm{e}}$ siècle. Il revient à Jacques Le Brun, sans doute l'un des meilleurs connaisseurs de la configuration mystique, de donner dans ce beau livre une "interprétation" du Pèlerin chérubinique, de son écriture/Écriture (die Schrift) pour inviter le lecteur à faire à son tour l'expérience à laquelle invite Angelus Silesius dans l'ultime distique (VI, 263) : «Ami, c'est assez. Au cas où tu voudrais lire plus, Alors va et deviens toi-même l'Écriture et toi-même l'essence ».

\section{AUTEURS}

\section{SOPHIE HOUDARD}

Université Sorbonne nouvelle. 